Monatsschr Kinderheilkd 2015 • 163:108-109

DOI 10.1007/s00112-014-3271-x

Online publiziert: 14. Februar 2015

(c) Springer-Verlag Berlin Heidelberg 2015

\title{
K.-M. Debatin
}

Universitätsklinik für Kinder- und Jugendmedizin, Ulm

\section{Nachsorge nach onkologischen Erkrankungen im Kindes- und Jugendalter}

Die Behandlung von Krebserkrankungen im Kindes- und Jugendalter ist eine der Erfolgsgeschichten der Onkologie. Noch vor wenigen Jahrzehnten gab es auch bei damals adäquater Therapie nur eine geringe Heilungschance. Inzwischen werden über $80 \%$ der Patienten dauerhaft geheilt. Einerseits zeigen die Tumor- und Leukämieerkrankungen im Kindes- und Jugendalter in der Regel eine aggressive Verlaufsform, andererseits macht gerade die $z$. T. hohe Proliferationsrate die Tumor- und Leukämiezellen sensibel für Chemotherapie und Bestrahlung. Die großen Erfolge wurden neben diesen zellbiologischen Besonderheiten insbesondere auch durch konzertierte Anstrengungen der pädiatrischen Onkologen erreicht, die über Jahre beispielhaft aufgezeigt haben, wie die Therapie durch kooperative Studienprotokolle Schritt für Schritt verbessert werden kann. Die dabei entwickelten Behandlungskonzepte sind in der Regel durch eine hochintensive, lang dauernde Therapie geprägt, die multimodal, je nach Tumorentität, Chemotherapie, Bestrahlung und chirurgisches Vorgehen kombiniert. Diese Therapien haben ein hohes akutes Toxizitätsrisiko aufgrund der akuten Nebenwirkung an Blutbildung und verschiedenen Organsystemen. In der Anfangszeit der Kombinationstherapien und multimodalen Therapiekonzepten in der pädiatrischen Onkologie hatte die Behandlung daher nicht nur eine hohe Morbidität, sondern auch eine relativ hohe Mortalität. Waren die Bemühungen der pädiatrischen Onkologen bis zum Ende des letzten Jahrhunderts vor allen Dingen dadurch geprägt, durch Risikostratifizierung und Identifizierung von Hochrisikogruppen unter Einsatz intensi- ver Therapiemaßnahmen überhaupt eine Heilung zu ermöglichen, stehen seit den 1990er Jahren und v. a. seit der Jahrtausendwende Überlegungen zur Reduktion von Toxizität im Vordergrund. Wenn der überwiegende Teil der Patienten geheilt werden kann, besteht die Aufgabe darin, dies mit der geringstmöglichen Toxizität zu erreichen. Der Fokus liegt dabei nicht mehr allein auf Akuttoxizität. Zahlreiche Studien belegen, dass Langzeitüberlebende ein gegenüber der Normalbevölkerung deutlich erhöhtes Risiko an therapiebedingten Langzeittoxizitäten haben. Dazu gehören zum einen ein erhöhtes Risiko für Zweittumorerkrankungen in direkter Assoziation mit der Therapie (lokale Bestrahlung, DNA-schädigende Chemotherapie) sowie Toxizitäten an verschiedenen Organsystemen, die sich wie die anthrazyklininduzierte Kardiomyopathie häufig erst in einer langen Latenz nach der Therapie manifestieren.

\section{》) Heilung der Patienten}

mit der geringstmöglichen Toxizität erreichen

Konsequenterweise hat die Nachsorge in der pädiatrischen Onkologie mit komplexen Nachsorgeprotokollen, die Bildgebung, Funktionsuntersuchungen und therapiespezifische Aspekte beinhalten, nicht nur den Fokus auf einer frühzeitigen Erfassung von Rezidiven, sondern auch die therapiebedingten Nebenwirkungen in einem besonderen Fokus. Daher hat die Gesellschaft für Pädiatrische Onkologie und Hämatologie (GPOH) die Arbeitsgruppe „Spätfolgen - Late Effects Surveillance System“ (LESS) etabliert, die 
sich umfassend mit der Registrierung von Langzeitkomplikationen und Toxizitäten sowie der Entwicklung zur standardisierten Nachsorge beschäftigt. Die Beiträge in diesem Heft spiegeln den aktuellen Stand der Überlegungen und Leitlinien dieser Diskussion wider.

Das Kernthema dieses Schwerpunkthefts der Monatsschrift Kinderheilkunde, die endokrinologische Nachsorge, wird im Beitrag von Denzer $u$. Wabitsch dargestellt. Für die strukturierte Nachsorge der endokrinologischen Langzeitfolgen hat auf Initiative der Autoren die Deutsche Gesellschaft für Kinderendokrinologie und Diabetologie (DGKED) gemeinsam mit der GPOH eine evidenzbasierte, deutschsprachige Leitlinie für die langfristige endokrinologische Nachsorge entwickelt.

Im Beitrag von Langer et al. wird generell das Konzept der Nachsorge nach onkologischen Erkrankungen dargestellt. Die Folgen, die noch im späten Erwachsenenalter auftreten können, reichen von direkt strahlenbedingten Toxizitäten wie Sekundärmalignomen bei Mediastinalbestrahlung (z. B. M. Hodgkin) über Nephrotoxizitäten aufgrund z. B. cisplatinbedingter Chemotherapie, Langzeithepatotoxizität, Knochennekrosen bei hochdosierter Kortisontherapie, insbesondere bei Heranwachsenden, Fertilitätsprobleme, Defizite der psychomotorischen Entwicklung nach Ganzhirnbestrahlung bis hin zu Langzeitstörungen an endokrinen Organen mit erheblichen Konsequenzen für die weitere Entwicklung und Lebensqualität. Der Beitrag zitiert entsprechende Daten zur Langzeitbeobachtung und unterstreicht die Bedeutung individueller Nachsorgepläne um Gesundheit und Lebensqualität nach der Behandlung von Krebserkrankungen. Angesichts der Tatsache, dass sich die derzeitige Gruppe der Überlebenden von Krebserkrankungen im Kindes- und Jugendalter von 30.000 jährlich um etwa 1500 erhöht, wird auch die soziale (und wirtschaftliche) Dimension deutlich.

Mit einer deutlich wahrzunehmenden Langzeitwirkung befasst sich der Beitrag von Müller et al. Sowohl durch lokale Bestrahlungen am wachsenden Skelettsystem oder, wie zuvor dargestellt, durch Schädelbestrahlung und durch Che- motherapie kommt es zum verminderten Körperwachstum. Auch die prophylaktische Schädelbestrahlung in mittleren Dosen, die noch lange zur Verhinderung eines Rezidivs im Zentralnervensystem (ZNS) bei Leukämien eingesetzt wurde, führt neben psychomotorischen Defiziten zu einer Störung des Wachstums. Darüber hinaus interferiert die antineoplastische Chemotherapie auf metabolischer Ebene mit verschiedenen Aspekten des Knochenstoffwechsels, die ebenfalls multiple Komplikationen wie Osteoporose und Osteonekrose nach sich ziehen können.

Neben Wachstumsstörungen sind Erkrankungen der Schilddrüse häufige Spätfolgen nach früherer antineoplastischer Therapie. Mit diesem Aspekt beschäftigt sich der Beitrag von Brabant et al. Die Störungen im Bereich der Schilddrüsen und Nebenschilddrüsen sind insbesondere auf Bestrahlungen der Halsregionen, z. B. im Rahmen eines M. Hodgkin, zurückzuführen. Erfassung und Behandlung der Erkrankungen an Schilddrüsen und Nebenschilddrüsen erfordern ein regelmäßiges Screening und eine spezifische (Substitutions-)Therapie.

$\mathrm{Zu}$ den häufigsten auch unmittelbar bemerkten Spätfolgen zählen die Störungen der Pubertätsentwicklung und eine beeinträchtigte Fertilität. Beide Spätfolgen schränken die Lebensqualität in erheblichem Umfang ein. Diese Aspekte der Langzeittoxizität werden im Beitrag von Denzer et al., unterlegt mit Daten aus großen Langzeitstudien, detailliert dargestellt. Neben dem Fokus auf der Gonadenfunktion werden Aspekte peripherer und zentraler Nebenniereninsuffizienz herausgearbeitet.

Zusammenfassend ist mit dem hohen Anteil an Langzeitüberlebenden nach Tumortherapie im Kindes- und Jugendalter für den pädiatrischen Onkologen eine neue Aufgabe herangewachsen. Die Sorge um Spätfolgen der Therapie steht gleichwertig neben dem Bemühen, mit allen zur Verfügung stehenden Mitteln eine Heilung zu erzielen. In langer Tradition haben die pädiatrischen Onkologen ihre Patienten über viele Jahre nachverfolgt, sodass wir verlässliche Daten über Langzeittoxizität besitzen. Wir haben die Aufgabe, für unsere Patienten auch langfristig eine adäquate medizinische Betreuung zu sichern. Dazu ist die Kooperation von Pädiatern mit den internistischen Kollegen in gemeinsamen Nachsorgesprechstunden erforderlich.

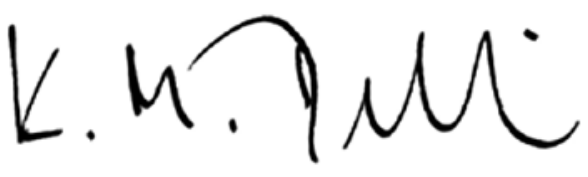

Prof. Dr. K.-M. Debatin

\section{Korrespondenzadresse}

Prof. Dr. K.-M. Debatin

Universitätsklinik für Kinder- und

Jugendmedizin

Eythstr. 24, $89075 \mathrm{UIm}$

klaus-michael.debatin@uniklinik-ulm.de

Interessenkonflikt. K.-M. Debatin gibt an, dass kein Interessenkonflikt besteht. 